Revue des patrimoines

$40 \mid 2019$

Lits historiques. Première anthologie des lits européens du XVe au XIXe siècle

\title{
Le lit du duc Antoine de Lorraine et de la duchesse Renée de Bourbon : une nouvelle vie grâce au numérique
}

The Bed of the Duke Antoine de Lorraine and the Duchesse Renée de Bourbon; a New Digital Life

\section{Muriel Barbier et Pierre-Hippolyte Pénet}

\section{(2) OpenEdition}

Journals

Édition électronique

URL : http://journals.openedition.org/insitu/24089

DOI : $10.4000 /$ insitu.24089

ISSN : 1630-7305

Éditeur

Ministère de la Culture

Référence électronique

Muriel Barbier et Pierre-Hippolyte Pénet, « Le lit du duc Antoine de Lorraine et de la duchesse Renée de Bourbon : une nouvelle vie grâce au numérique », In Situ [En ligne], 40 | 2019, mis en ligne le 15 septembre 2019, consulté le 04 octobre 2019. URL : http://journals.openedition.org/insitu/24089 ; DOI : 10.4000/insitu.24089

Ce document a été généré automatiquement le 4 octobre 2019.

\section{c) $(9)$}

In Situ Revues des patrimoines est mis à disposition selon les termes de la licence Creative Commons Attribution - Pas d'Utilisation Commerciale - Pas de Modification 4.0 International. 


\section{Le lit du duc Antoine de Lorraine et de la duchesse Renée de Bourbon : une nouvelle vie grâce au numérique}

The Bed of the Duke Antoine de Lorraine and the Duchesse Renée de Bourbon; a New Digital Life

Muriel Barbier et Pierre-Hippolyte Pénet

\section{Une commande de la Cour de Lorraine}

À l'aube $\mathrm{du} \mathrm{xVI}^{\mathrm{e}}$ siècle, les duchés de Lorraine et de Bar constituent un territoire souverain, indépendant du royaume de France. En 1480, le duc René II de Lorraine, vainqueur du duc de Bourgogne Charles le Téméraire lors de la bataille de Nancy trois ans auparavant, réunit sous sa tutelle les deux duchés, devenant ainsi duc de Lorraine et de Bar. En 1489, son épouse, la duchesse Philippe de Gueldre, met au monde un fils prénommé Antoine. Le jeune prince passe son enfance au palais ducal de Nancy puis est envoyé parfaire son éducation à la Cour de France, où il se lie d'amitié avec le duc d'Angoulême, futur François I ${ }^{\text {er }}$, qui n'a que cinq ans de moins que lui. Devenu duc (fig. 1) à la mort de son père en 1508, le jeune souverain participe aux côtés des Français aux campagnes d'Italie et s'illustre en particulier lors des batailles d'Agnadel en 1509 et de Marignan en 1515. Cette même année, il assiste au sacre du roi François I ${ }^{\text {er }}$ et épouse, le 26 juin, au château d'Amboise, Renée de Bourbon, sœur de Charles III de Bourbon, fait connétable par le roi quelques mois plus tard ${ }^{1}$. 
Figure 1

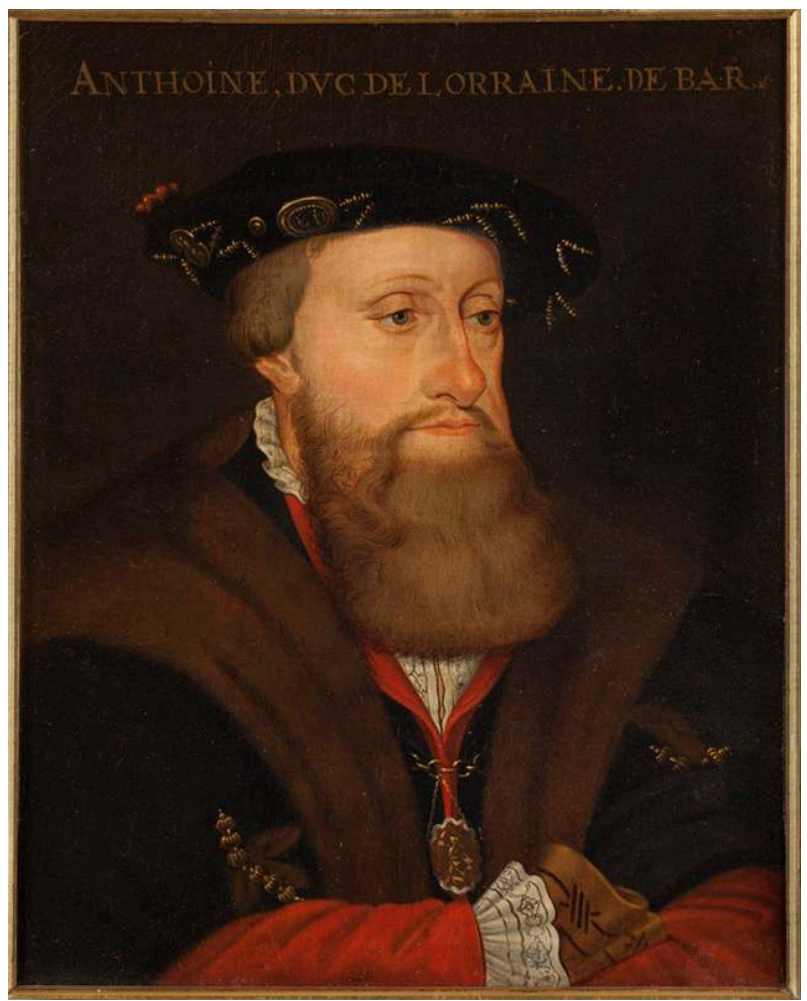

D'après Hugues de La Faye, Anthoine duc de Lorraine de Bar, XIXe siècle, peinture à I'huile sur bois. Nancy, Palais des ducs de Lorraine-Musée lorrain, 95.337.

(C) Michel Bourguet.

2 Le souvenir des deux jeunes époux peut être aujourd'hui évoqué grâce à un meuble exceptionnel, conservé au Palais des ducs de Lorraine-Musée lorrain, considéré comme le plus ancien lit princier conservé en France à ce jour (fig. 2). Aucune source archivistique ne permet de préciser les circonstances exactes de sa commande ni les noms des artisans ayant œuvré à sa réalisation mais il est fort probable qu'il a été exécuté l'année du mariage ducal. Le meuble est cité pour la première fois en 1524 lors du baptême du prince Nicolas au château de Bar-le-Duc, principale résidence ducale avec le palais nancéien. Dans sa relation de l'événement, l'historiographe Nicolas Volcyr (v. 1480-1541) décrit en effet avec précision l'ameublement de la demeure. Il mentionne dans la chambre de la duchesse, "richement parée » : «le ciel tout de mesme et semblable artifice, et le charlict faict de menuserie subtille et ingenieuse en suivant pareillement le traict et gect du paintre, dont le bois estoit doré par-dessus la taille et scrupture de fin or, et figure comme la susdite tapicerie, avec tant de ces belles devises : J'espere avoir, Ung pour jamais [...] $»^{2}$. Le lit apparaît ensuite, de manière parfois fragmentaire, dans trois inventaires du mobilier du palais de Nancy en 1532, 1543 et $1606^{3}$. Après cette dernière mention, il quitte le palais ducal à une date inconnue avant de réapparaître au XIX ${ }^{\mathrm{e}}$ siècle. 


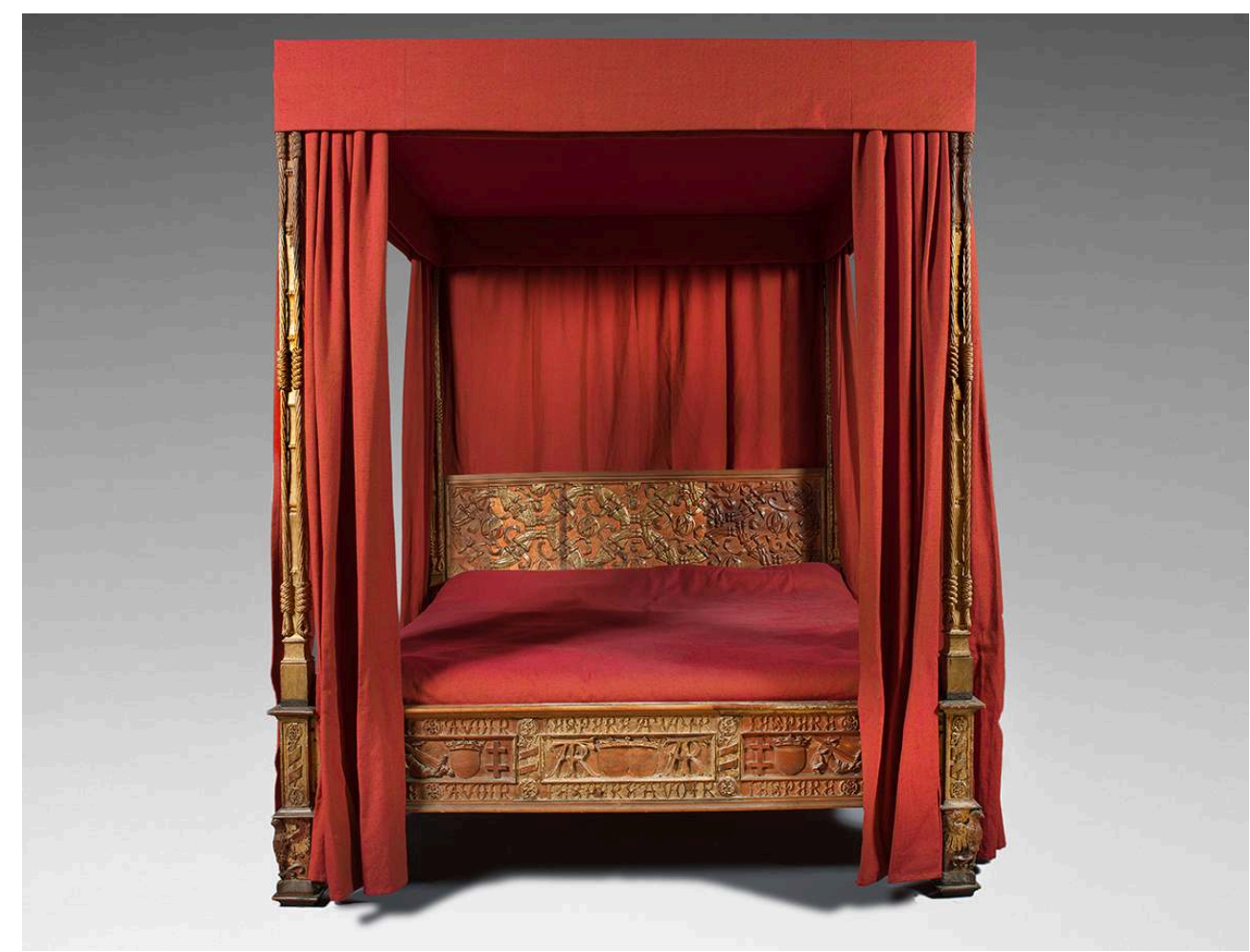

Lit du duc Antoine de Lorraine et de Renée de Bourbon, 1515, bois sculpté, doré et peint. Nancy, Palais des ducs de Lorraine-Musée lorrain, dépôt du Mobilier national, D.95.255.

(c) Philippe Caron.

\section{La redécouverte du lit}

3 Si l'on en croit la version de l'historien lotharingiste Prosper Guerrier de Dumast ${ }^{4}$, le lit ducal aurait été redécouvert dans les années 1820 par un brocanteur de Nancy dans les ruines du château de Vaudémont, " entre le fond d'une vieille maison et l'un des rares pans» subsistant de l'ancienne forteresse (fig. 3). Fustigeant l'indifférence des Lorrains, l'historien déplore l'envoi de cette "relique touchante » à Paris «chez les marchands de bric-à-brac », tout en attribuant par erreur les devises apparaissant sur le lit au duc René II et non à son fils Antoine ${ }^{5}$. Si la description de Guerrier de Dumast semble assez romanesque, il est attesté que le lit entre en possession d'un jeune archéologue en 1835. Ernest-Louis-Hippolyte-Théodore Grille de Beuzelin ${ }^{6}$, membre de la Société des antiquaires de France, chargé par Guizot de l'élaboration d'une Statistique monumentale de la France, est en effet envoyé en mission cette année-là pour visiter les arrondissements de Nancy et de Toul ${ }^{7}$. Il est donc très vraisemblable que ce dernier découvre ou achète le lit à cette occasion et décide de le rapporter à Paris. 
Figure 3

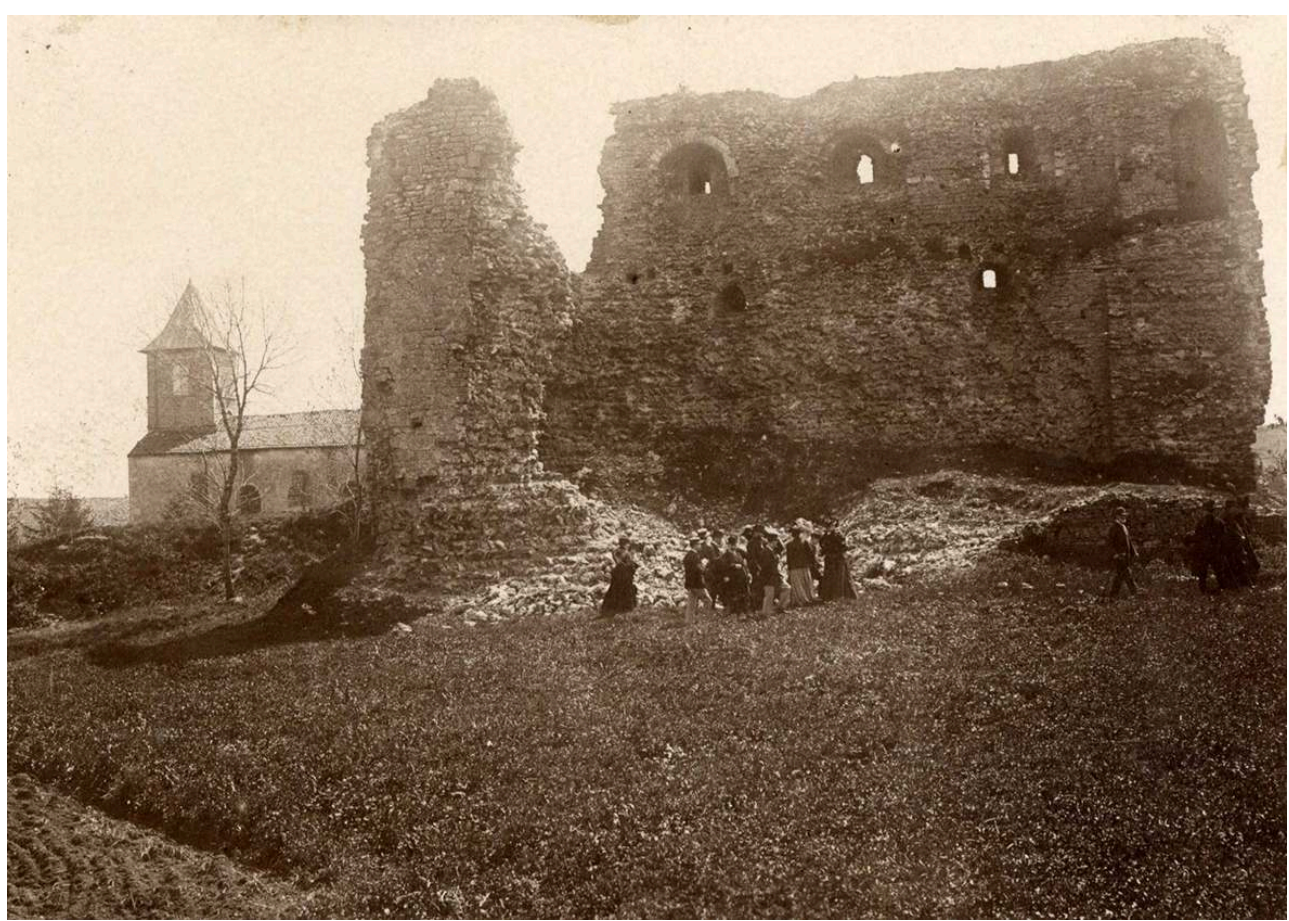

Vue des ruines du château de Vaudémont, 1897, photographie. Nancy, Palais des ducs de LorraineMusée lorrain, 2013.0.1926.

(c) Musée lorrain.

Le 31 mars 1836, le meuble apparaît en effet pour la première fois dans un acte officiel de vente conclu entre Grille de Beuzelin et le Mobilier de la Couronne. Décrit comme « un lit en bois sculpté anciennement peint et doré, orné de blasons de Lorraine, de Montpensier et de Bourbon, ainsi que des devises des maisons de Lorraine et de Montpensier. Longr 6 pds largr 4 pds environ. Prix $800 \mathrm{fr} .{ }^{8}$, le lit est enregistré au journal du Garde-Meuble le 26 avril sous le numéro 13569 avec une description identique $^{9}$. Dans le contexte de remeublement de plusieurs résidences royales voulu par Louis-Philippe, on songe à l'envoyer soit au palais de Fontainebleau soit au palais de $\mathrm{Pau}^{10}$. Dans cette optique, le meuble est restauré en 1842, agrandi et modifié ainsi que l'indique sa réinscription au journal du Garde-Meuble, sous le numéro 33008, le $1^{\text {er }}$ décembre : «Une couchette en bois sculpté ornée des blasons et devises des maisons de Lorraine et Montpensier et de Bourbon, montants à jour entourés de cordes sculptés à jour, dossier, à panneaux sculptés. Hr 2,62 Lr 2,25 largr 2,40. $1343 \mathrm{fr}$ » $^{11}$. Lors de cette restauration, un soin particulier est accordé à rendre visible la différenciation entre les parties d'origine et les parties modernes, ces dernières ne recevant ni peinture ni dorure (fig. 4). 
Figure 4

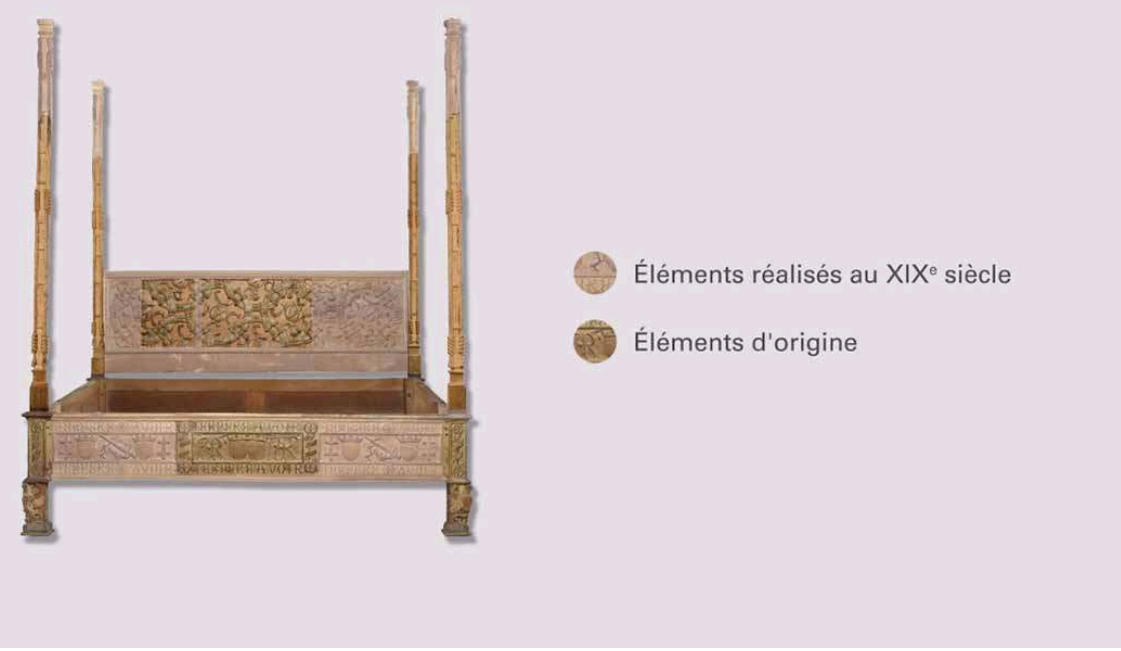

Schéma distinguant les parties d'origine du lit (en surbrillance) et les parties rajoutées au xIx ${ }^{e}$ siècle. (c) Mazédia/Ludovic Gicqueau, 2016.

5 En juillet 1852, Prosper Guerrier de Dumast et Alexandre Gény, tous deux membres de la Société d'archéologie lorraine fondée quatre ans auparavant ${ }^{12}$, alertent leurs confrères sur la présence du lit à Paris et proposent d'entamer des démarches afin que ce témoignage insigne des ducs de Lorraine puisse être déposé au tout récent Musée lorrain inauguré en $1850^{13}$. Ce n'est toutefois qu'en 1872 que la $\mathrm{III}{ }^{\mathrm{e}}$ République accède à la demande de la Société grâce à l'appui de deux députés, Étienne-Auguste Ancelon et Henri Varroy. Un dessin représentant le meuble, conservé à Nancy, témoigne probablement de cette entreprise (fig. 5). 


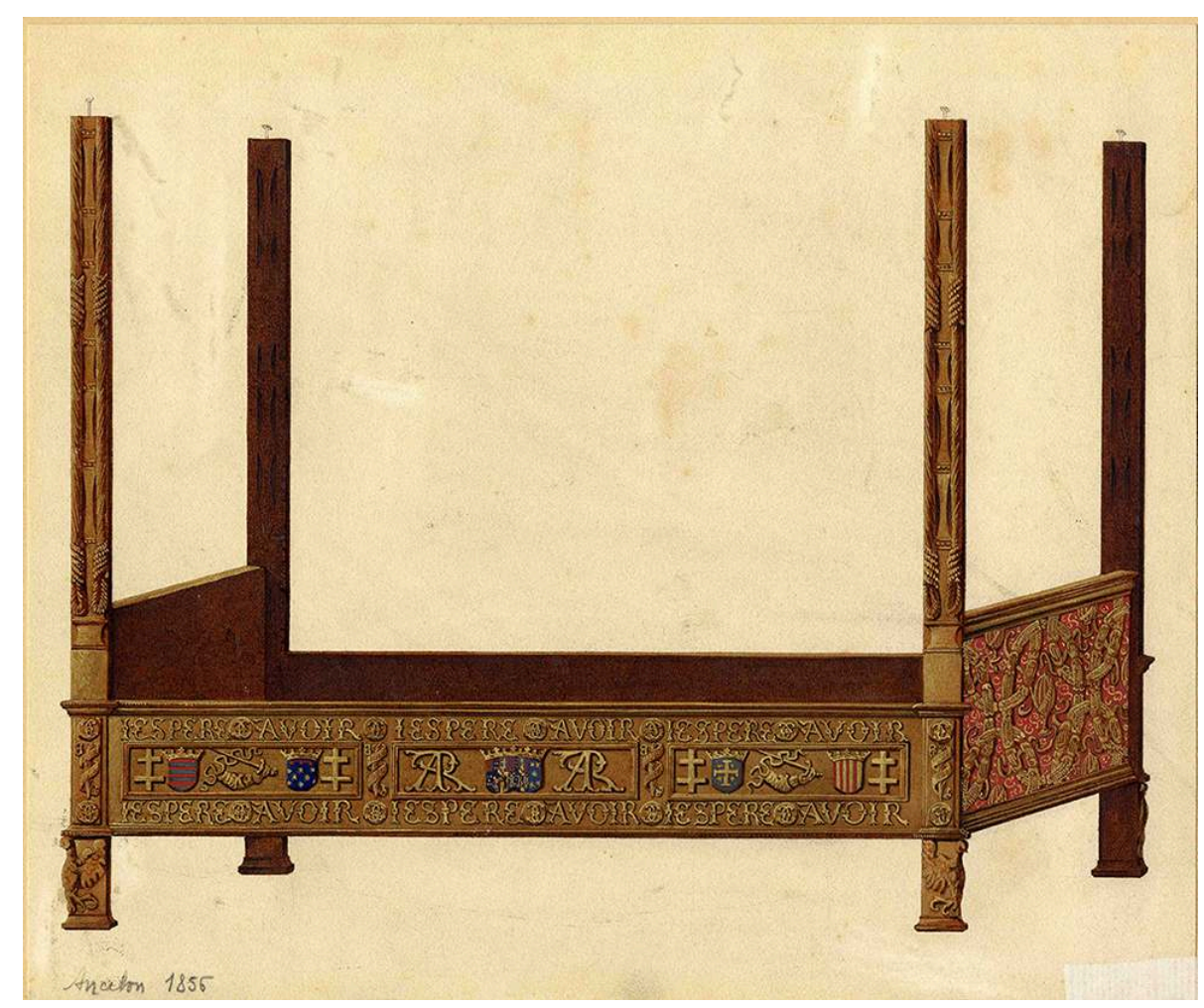

Lit du duc Antoine de Lorraine et de Renée de Bourbon, 1856, dessin à la gouache et au crayon. Nancy, Palais des ducs de Lorraine-Musée lorrain, 2013.0.1974.

(C) Musée lorrain.

La mention au crayon «Ancelon, 1856 » désigne le médecin lorrain et membre de la Société d'archéologie lorraine avant qu'il ne devienne député de Meurthe-et-Moselle (en 1871). On ignore néanmoins si ce dernier est l'auteur ou le donateur du dessin qui semble proposer une hypothèse de restitution ou bien un relevé exact du lit à partir des seuls éléments d'origine retrouvés ${ }^{14}$. En juillet 1872, on peut lire dans le Journal de la Société d'archéologie lorraine: "Après bien des démarches, restées longtemps infructueuses, le Musée lorrain vient enfin - grâce à l'intervention de deux de nos honorables députés, MM. Ancelon et Varroy, - de s'enrichir d'un objet dont la place était naturellement marquée sous les voûtes du palais ducal de Nancy. C'est le lit du duc Antoine, morceau précieux à la fois comme œuvre d'art et comme souvenir historique ${ }^{15}$." La "précieuse » relique, dotée d'une parure textile moderne, est installée dans une salle du musée que l'architecte Émile Boeswillwald vient d'établir à l'extrémité de la galerie des Cerfs, ancien espace d'apparat du palais ${ }^{16}$. Ne correspondant pas historiquement aux appartements du duc Antoine qui se trouvaient dans l'aile est de la demeure, cette pièce est aménagée dans un esprit de period room avant l'heure. Le lit est notamment entouré de la tenture de la Condamnation de Banquet, commandée par le duc Antoine aux ateliers tournaisiens, d'une cheminée du Xvi siècle provenant de Saint-Nicolas-de-Port, offerte en 1871 au musée par le préfet Fernand de Montesquiou Fezensac, et surmonté d'un plafond "à la française » aux poutres ornées de peintures néo-Renaissance représentant les emblèmes lorrains (fig. 6). Avec la tenture de Tournai, le lit constitue aujourd'hui le seul vestige de l'ameublement du palais ducal de Nancy du Xvi ${ }^{\mathrm{e}}$ siècle, le reste du mobilier ayant progressivement disparu 
au XVII ${ }^{\mathrm{e}}$ siècle, dans le contexte des occupations militaires françaises successives, puis au XVIII ${ }^{e}$ siècle, lors du départ définitif de la famille ducale pour l'Italie puis l'Autriche ${ }^{17}$.

Figure 6

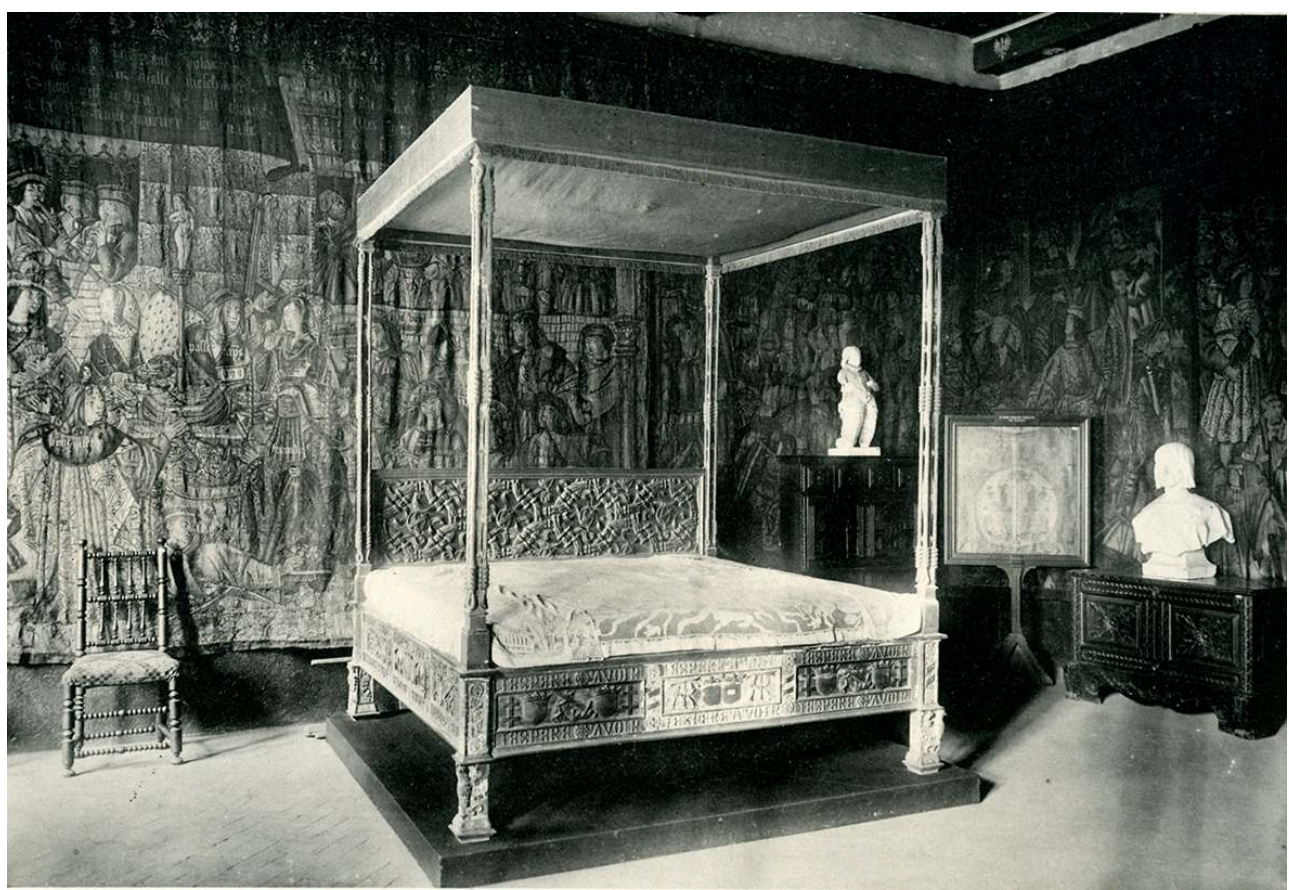

Royer, Lit du duc Antoine au Musée lorrain, 1896, photographie. Nancy, Palais des ducs de LorraineMusée lorrain, 2013.0.1969.

(c) Musée lorrain.

\section{Un meuble d'apparat}

$7 \quad$ Les descriptions anciennes des éléments textiles aujourd'hui disparus et l'observation des éléments sculptés subsistants attestent de la fonction de représentation d'un tel meuble dont la décoration met en exergue ses illustres propriétaires. Héraldique et emblématique célèbrent conjointement le prestige ducal et l'union des deux époux.

8 Sur la traverse centrale apparaissent leurs deux écus accolés surmontés de la couronne ducale. Les armes du duc Antoine sont constituées des blasons des quatre royaumes de Hongrie, de Sicile, de Jérusalem et d'Aragon, prétentions héritées de René Ir d'Anjou, et de ceux des trois duchés d'Anjou, de Bar et de Lorraine. L'écu de la duchesse est parti des armes de son mari et des armes de la maison de Bourbon. Les quatre royaumes étant les plus prestigieux, ils sont également reproduits séparément dans les panneaux latéraux. De chaque côté des deux écus accolés sont représentés les monogrammes des deux époux fusionnés en un seul élément (fig. 7). 
Figure 7

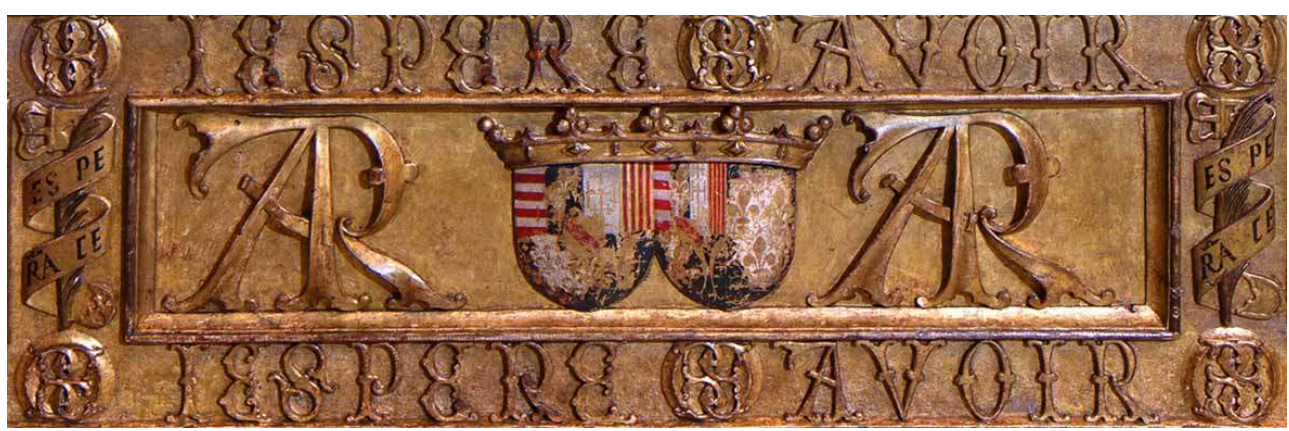

Lit du duc Antoine de Lorraine et de Renée de Bourbon (détail de la traverse centrale).

(c) Michel Bourguet.

9 La part la plus importante de la décoration est consacrée à l'emblématique. Dans les traverses latérales, on distingue tout d'abord le bras armé ou dextrochère (fig. 8). Choisi par le duc René II qui le transmet à son fils, ce bras armé symbolise la puissance divine. Il est associé à cet extrait du Magnificat : «Fecit potentiam in brachio suo » (il a placé la puissance dans son bras). Par extension, il désigne également la puissance du duc et fait écho à la victoire de René II contre Charles le Téméraire à la bataille de Nancy en 1477. Le second emblème lorrain est la croix dite "de Lorraine » à deux traverses, héritage de la famille d'Anjou, qui place le duc de Lorraine comme défenseur de la foi catholique. Enfin, un troisième emblème lorrain particulièrement important apparaît sur les pieds du lit: il s'agit de l'alérion, petit aigle normalement représenté sans bec ni pattes, qui figure dans les armes du duché de Lorraine ${ }^{18}$.

Figure 8

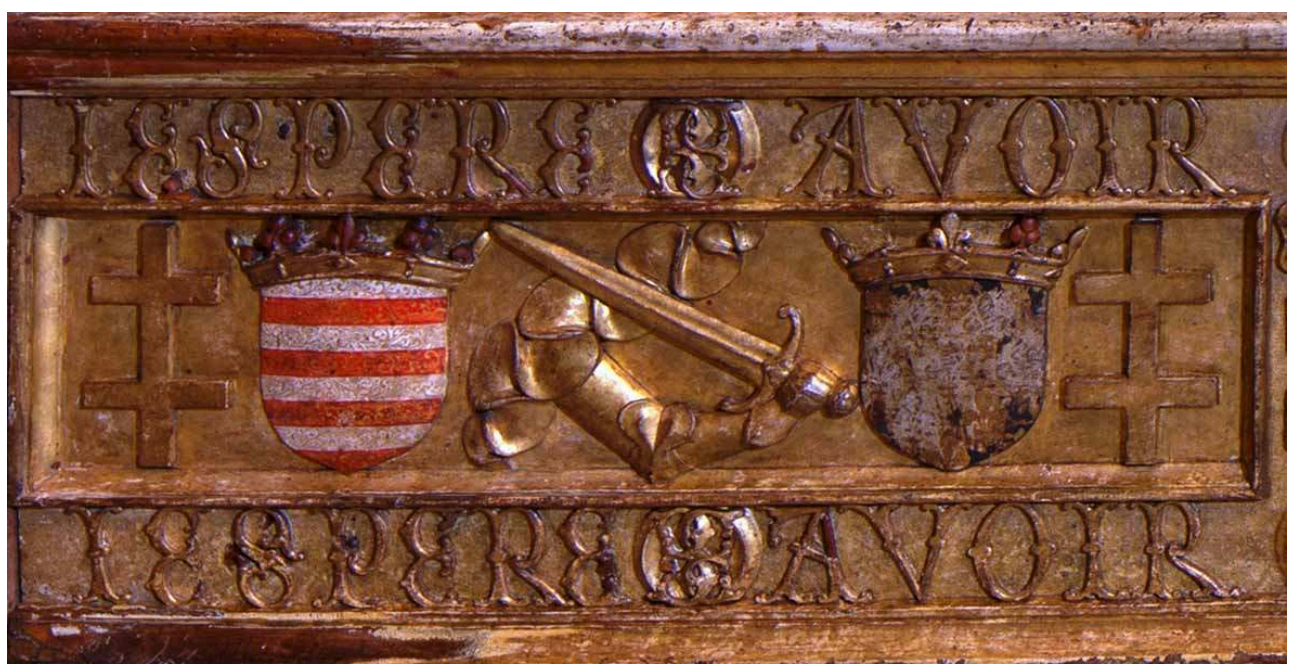

Lit du duc Antoine de Lorraine et de Renée de Bourbon (détail d'une dextrochère).

(c) Musée lorrain.

Aux côtés de ces emblèmes lorrains, les traverses du lit sont ornées des devises du couple ducal. Choisie par Louis II de Bourbon en 1633, «Espérance» est celle de la maison de Bourbon. Elle apparait chargée sur une ceinture de couleur bleue, tout à la fois symbole de lien et évocation de la ceinture de la Vierge. Cette ceinture s'enroule ici autour d'une palme de victoire. Faisant écho à celle de la famille de son épouse, la 
devise du duc Antoine «J'espère avoir » figure également sur les traverses du lit ainsi que sur le dosseret dans des phylactères de couleur bleue. Adoptée à une période contemporaine de la guerre des Rustauds et illustrant les entreprises d'Antoine au service de la catholicité, cette devise prend ici une signification multiple, à la fois politique, religieuse et matrimoniale. Faisant le lien avec ces deux premières devises, des $\mathrm{E}$ s'entrecroisent en signe d'union. On distingue également une troisième devise choisie par la duchesse : «Ung pour jamais » qui, de la même manière, semble tout à la fois désigner la fidélité conjugale et, selon Nicolas Volcyr, la dévotion de la duchesse en un Dieu unique ${ }^{19}$.

11 Sur le dosseret à fond rouge orné de petits motifs floraux (fig. 9), ces devises sont accompagnées de deux motifs. Le premier est la sphère armillaire, emblème personnel choisi par le duc Antoine comme un rébus. $\mathrm{Au} \mathrm{XvI}{ }^{\mathrm{e}}$ siècle, le terme "sphère » est en effet prononcé "espère », ce qui fait directement référence à la devise "J'espère avoir ». L'emblème représente également la perpétuité de l'union entre les deux époux. Le second emblème est un chapeau végétal de triomphe qui évoque ici les couronnes de fleurs portées par les mariées le jour des noces. Il a la spécificité d'être lié par des fois, mains jointes symbolisant précisément la foi échangée par les époux qui font ce geste lors de la cérémonie. Enfin, un dernier emblème peut être repéré dans l'ornementation des colonnes du lit, formée de cordelières ornées de trois nœuds, qui évoquent peutêtre les Cordeliers desservant la nécropole familiale.

Figure 9

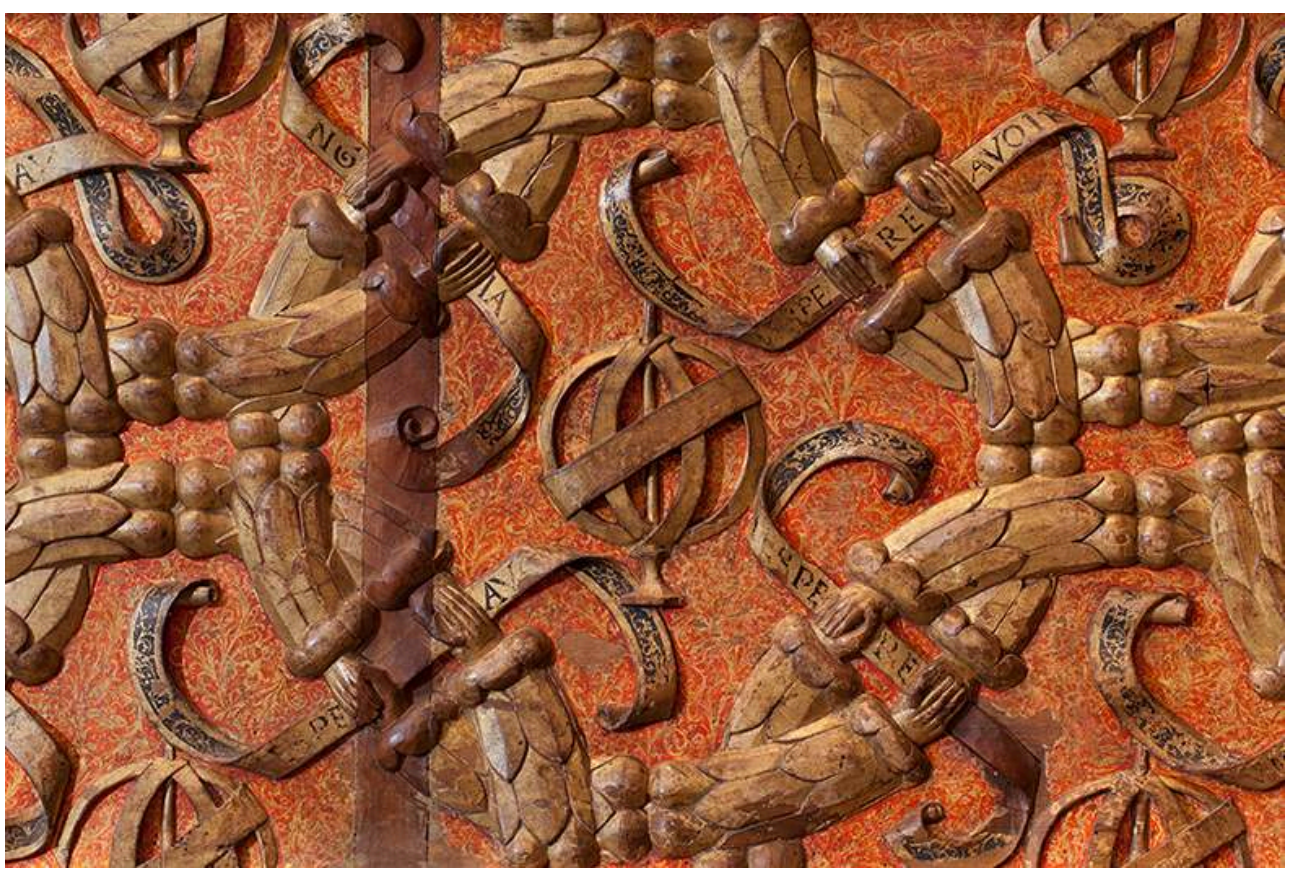

Lit du duc Antoine de Lorraine et de Renée de Bourbon (détail du dosseret).

(c) Michel Bourguet.

\section{Trois états de la parure textile}

12 Les trois inventaires publiés en 1891 livrent de précieuses informations sur les éléments textiles ayant composé ce lit durant les règnes des ducs de Lorraine Antoine 
(1508-1544) et Charles III (1545-1608). Bien que les dimensions des pièces d'étoffe n'y soient pas reportées, ces documents permettent néanmoins d'en comprendre l'aspect.

La réflexion sur la parure textile du lit a débuté en 2012 alors que le Musée lorrain préparait l'exposition "Un Nouveau monde. Naissance de la Lorraine moderne ${ }^{20}$. Le châlit ayant été remonté pour l'occasion, il paraissait essentiel de le doter d'étoffes pour en restituer le volume global et montrer au visiteur l'importance de l'apparat textile sur les lits du xvI ${ }^{\mathrm{e}}$ siècle. L'ambition initiale était de restituer la parure textile telle qu'elle pouvait être comprise à la lecture des sources. La bibliographie existante sur le meuble se révèle pourtant décevante sur ce point et insiste surtout sur la signification héraldique des ornements brodés ${ }^{21}$. Le comité scientifique ${ }^{22}$ réuni pour ce projet s'est rapidement trouvé confronté à deux difficultés majeures: d'une part, l'absence de descriptif ancien suffisamment précis pour permettre la recréation à l'identique de la parure de 1532 et, d'autre part, le coût potentiel d'une telle restitution. Sur les bases des réflexions menées au sein du Comité lit, notamment en termes de proportions, la parure proposée donne à voir l'habillage du bois et le coloris dominant : le cramoisi. Le choix s'est arrêté sur une étoffe du commerce approchante au tombé satisfaisant en sergé de lin couleur brique ${ }^{23}$.

14 L'importante campagne de développement des supports de médiation dans le cadre du projet de rénovation et d'extension du Palais des ducs de Lorraine-Musée lorrain a ouvert de nouvelles perspectives. Les chefs-d'œuvre des collections du musée - dont le lit - ont été dotés d'outils multimédia permettant une nouvelle approche plus contextualisée. L'un des objectifs principaux du dispositif dédié au lit du duc Antoine et de la duchesse Renée de Bourbon était de proposer la restitution numérique de la garniture de 1532 mais aussi des deux suivantes, 1543 et $1606^{24}$.

La recherche d'éléments utiles pour les graphistes et informaticiens s'est faite à partir de trois sources : les archives et la documentation écrite susmentionnée, les documents iconographiques (peintures, enluminures, etc.) et les collections d'étoffes anciennes conservées. La part d'interprétation étant large dans ce type d'exercice, les résultats de ce travail entre infographistes et conservateurs ont été soumis à plusieurs reprises aux membres du Comité lit pour validation ${ }^{25}$.

parure de ce lit impliquait d'en restituer les volumes, les proportions et les dimensions, mais aussi les techniques d'accrochage des étoffes sur le bâti et enfin l'aspect des armures et des ennoblissements textiles. Le projet étant de s'approcher au plus près des volumes du lit au Xvi ${ }^{\mathrm{e}}$ siècle, il n'était pas envisageable de s'appuyer sur les dimensions actuelles du meuble, modifié et agrandi dans les années $1840^{26}$. C'est donc à la lecture d'autres inventaires contemporains décrivant des "lits d'étoffe ", en particulier celui du garde-meuble de François $I^{\text {er }}$ daté de $1542^{27}$, qu'une proposition de volumes a été faite. Les indications portées dans cet inventaire ont permis de définir la hauteur des courtines, celle des pentes, ainsi que la largeur et la longueur de la couche. Les proportions ainsi obtenues sont plus réduites modestes, comparées au lit actuel.

17 En raison de leur absence dans les inventaires des biens des ducs de Lorraine aux numéros correspondant à ce lit, et suite aux échanges avec les membres du Comité lit, nous avons choisi de ne pas restituer virtuellement un traversin et des oreillers qui figurent généralement sur les lits d'apparat. Néanmoins, un travail de recherche avait été préalablement mené dans la perspective de leur éventuelle restitution. Le résultat de ce travail apparaît dans le multimédia. Pour le volume des carreaux (coussins) ${ }^{28}$, le 
modèle de référence retenu est celui que l'on peut voir dans La Naissance de l'Amour, tableau du milieu du XvI ${ }^{e}$ siècle attribué au Maître de Flore conservé au Metropolitan Museum of Art de New York (fig. 10) ${ }^{29}$.

Figure 10

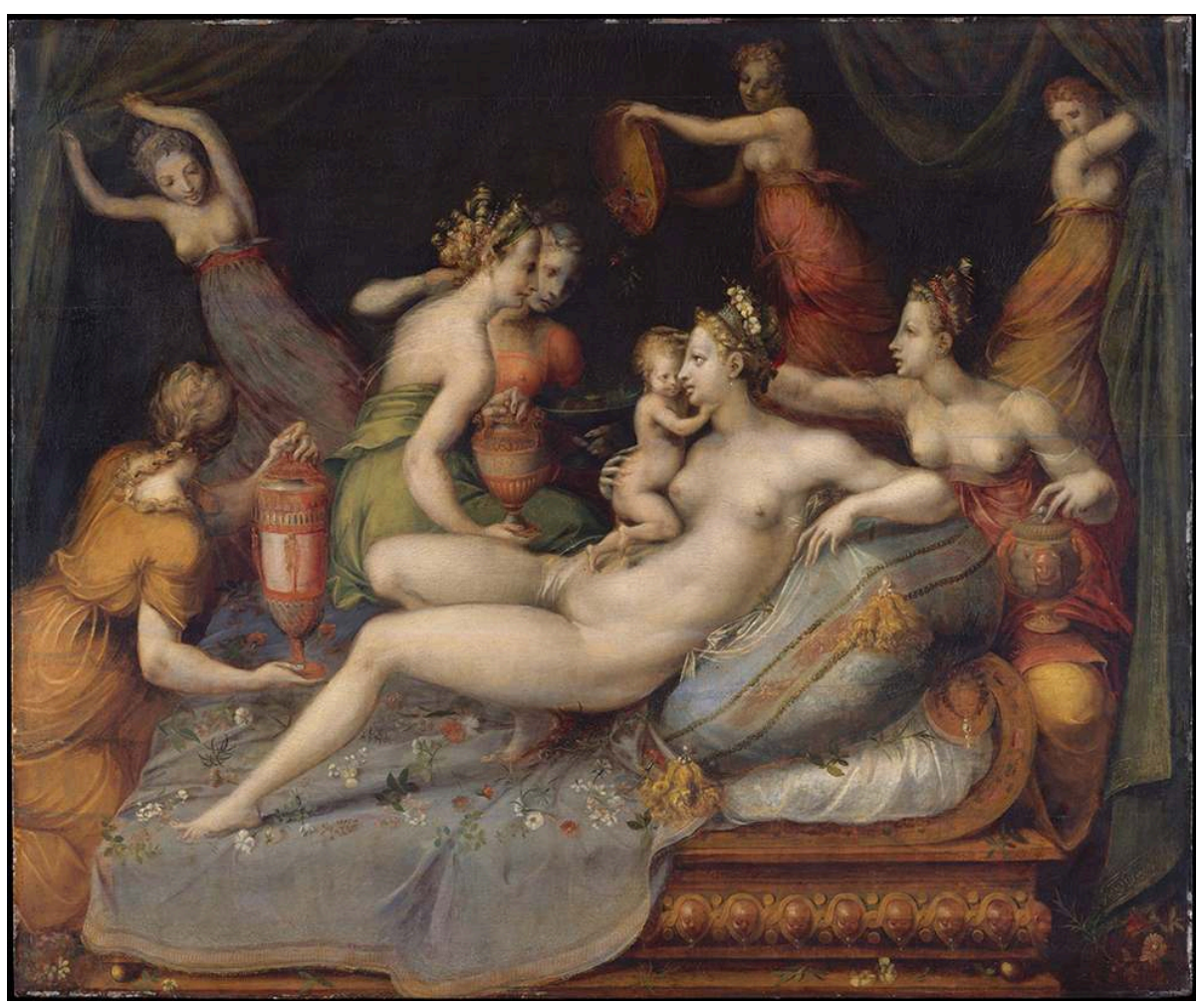

Maître de Flore, La Naissance de l'Amour, $2^{\mathrm{e}}$ moitié du xvle siècle, peinture à l'huile sur bois, New York, Metropolitan Museum of Art, 41.48. Creative commons (No Copyright). Base du Metropolitan Museum of Art : https://www.metmuseum.org/art/collection/search/437006? searchField=ArtistCulture\&amp;sortBy=relevance\&amp;ft=Master+Flora\&amp;offset=0\&amp;rpp=20\&amp;pos=1

L'épaisseur de la couche (induite par le nombre de matelas superposés), la hauteur des courtines (en particulier leur distance par rapport au sol) ainsi que la hauteur des pentes ont fait l'objet de validations par le Comité lit ${ }^{30}$ (fig. 11). Tous ces éléments établis, il fallut envisager la restitution virtuelle de leur mode d'accrochage sur le bâti. Bien que ce détail n'apparaisse pas dans les sources, mais compte tenu de la richesse du lit ducal, nous avons pensé qu'il était pourvu de doubles pentes, c'est-à-dire de pentes extérieures et de pentes intérieures qui dissimulaient le système de tringles et d'anneaux nécessaire à la suspension des courtines. Cette étape a donné lieu à un schéma préparatoire à la modélisation (fig. 12). 
Figure 11

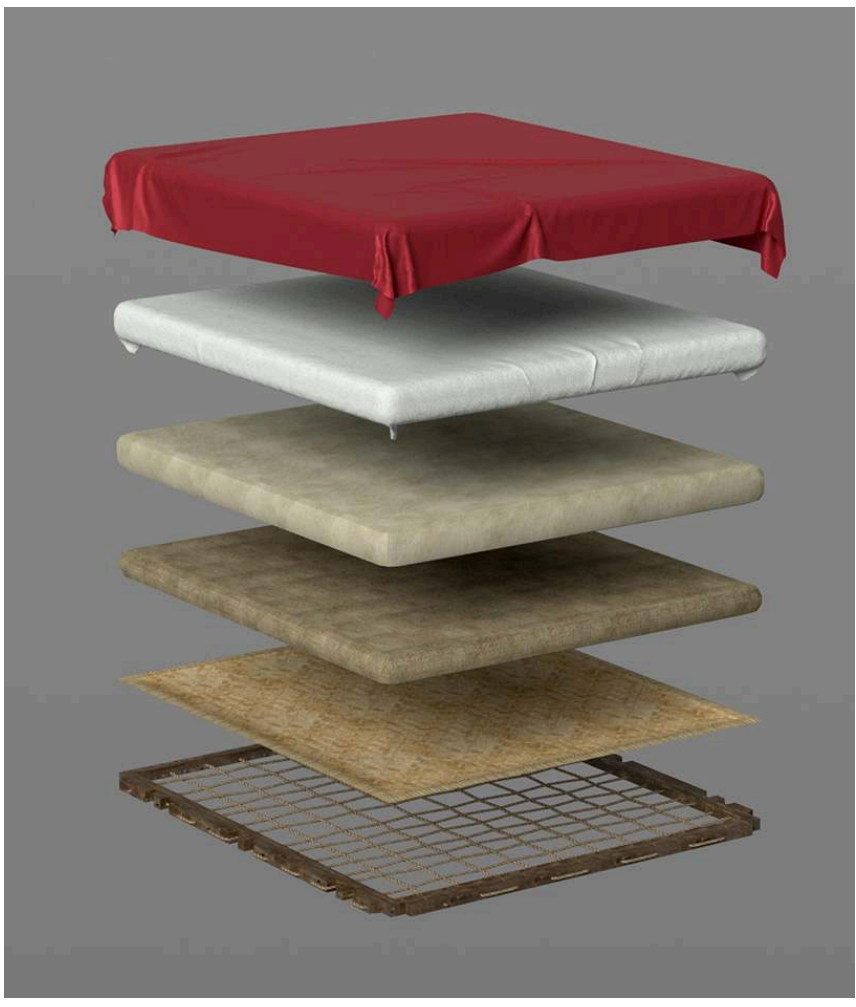

Superposition des éléments de literie, d'après Muriel Barbier.

(c) Mazédia/Ludovic Gicqueau, 2016.

Figure 12

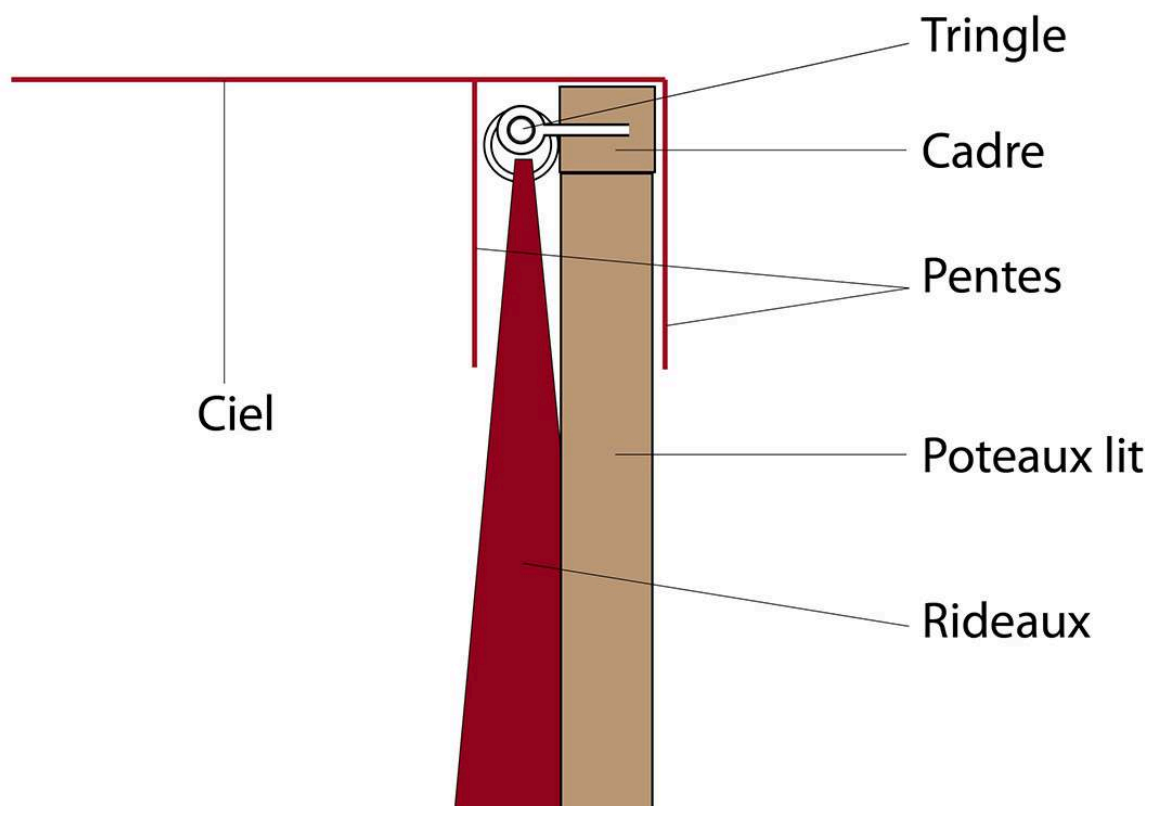

Schéma de montage des courtines et des pentes sur le bâti en bois, d'après Muriel Barbier.

(c) Mazédia/Ludovic Gicqueau, 2016. 
19 La partie la plus complexe de ce travail de restitution virtuelle concernait probablement les textiles en raison de la diversité des armures utilisées, des techniques de broderie mais aussi des effets de la lumière sur ces matériaux ${ }^{31}$. Tout au long des échanges avec le prestataire, le principe retenu a été de toujours revenir aux inventaires et de décrypter les indications qu'ils contenaient pour proposer des textiles approchants et des mises en forme pertinentes.

\section{L'état de 1532}

"dosseret", qui désigne le grand pan d'étoffe situé au niveau de la tête du lit et couvrant toute la hauteur entre le ciel et la couche, et, d'autre part, le «ciel», substructure portée par les colonnes (ou quenouilles) ou suspendue au plafond audessus de la couche ${ }^{33}$. Chargé d'une symbolique forte liée à la pourpre impériale, le cramoisi était aussi l'une des couleurs les plus coûteuses à obtenir; il était fréquemment employé pour les lits d'apparat ${ }^{34}$. Dosseret et ciel sont tous deux en satin, étoffe dont la densité des liages les fait disparaitre dans le fond et produit l'aspect lisse et brillant qui le caractérise. Le satin peut être tissé avec différents matériaux, mais il est vraisemblable qu'il s'agisse ici d'un satin de soie. Le dosseret associe satin et velours damassé ; nous pensons que ce dernier terme désigne en réalité un velours de soie ciselé, c'est-à-dire un velours aux motifs donnant l'effet d'un damas. Les motifs décrits reprennent manifestement ceux sculptés et peints sur les bois. Pourtant, ce document de 1532 n'aide pas à comprendre si les chapeaux de triomphe, phylactères, devises et sphères étaient tissés ou brodés. C'est la description précédemment évoquée donnée par Nicolas Volcyr lors du baptême du prince Nicolas qui rend la technique du décor perceptible. Cette description nous apprend que le décor de la chambre de la duchesse (tentures et lit) est : « faicts de satin cramoisy semez par tout de speres et chapeaux de festes transversez et entrelassez de ceintures et rolleaux faictz et tirrez à l'esguille par le brodeux subtil, aguz et fort ingenieux $[. ..]{ }^{35}$. Les emblèmes et devises étaient donc brodés à l'aiguille.

24 Les courtines (ou rideaux) ne sont pas mentionnées. Nous avons donc été arrêté notre choix sur des courtines en velours ciselé rouge qui semblaient les plus vraisemblables. Ce velours a été modélisé à partir d'un lé de velours conservé au Victoria and Albert Museum de Londres qui était pertinent quant aux motifs pour la période concernée (fig. 13). 
Figure 13

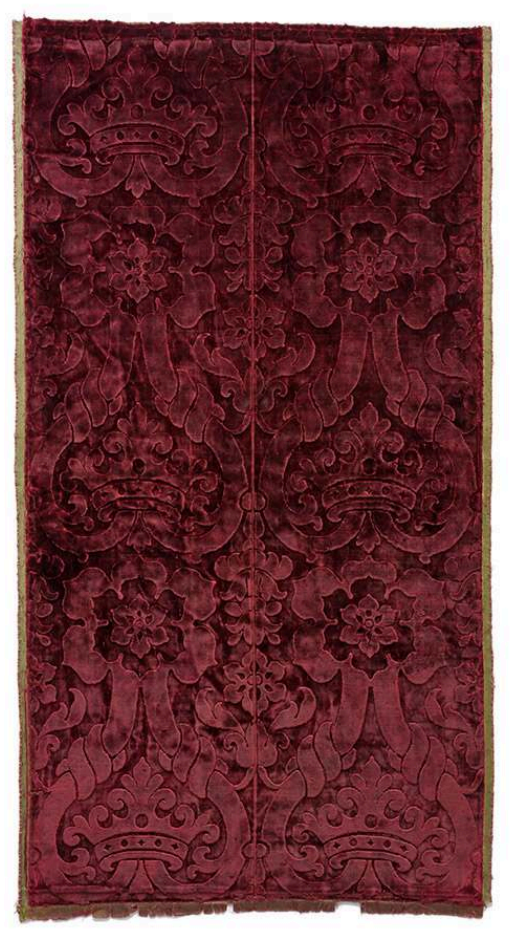

Venise, fragment d'étoffe, deuxième quart du XVIe siècle, velours de soie ciselé. Londres, Victoria and Albert Museum, 587-1892.

(C) Victoria and Albert Museum, London. Base du Victoria and Albert Museum : http:// collections.vam.ac.uk/item/0137412/panel-unknown/.

Pour le satin, le travail des informaticiens a été facilité grâce à l'existence dans le commerce de satins de soie unis, aisés à modéliser. Un des principaux enjeux résidait dans le rendu des broderies de filés or et de filés argent. Si le chevet sculpté donnait un exemple des motifs, il fallut s'appliquer à restituer le relief de la broderie à l'aiguille sur le satin et la matière des fils de soie et des fils métalliques. Après plusieurs essais, un équilibre a été trouvé entre les proportions des différents emblèmes, l'épaisseur des broderies et les coloris des fils comme du fond (fig. 14). Une sélection de fragments textiles du musée national de la Renaissance de couleur cramoisie et enrichis de flottés or et argent ont servi de modèles pour le contraste des broderies sur le satin cramoisi ; ils ont permis d'aboutir à une proposition de restitution pour un premier état du lit (fig. 15). 
Figure 14

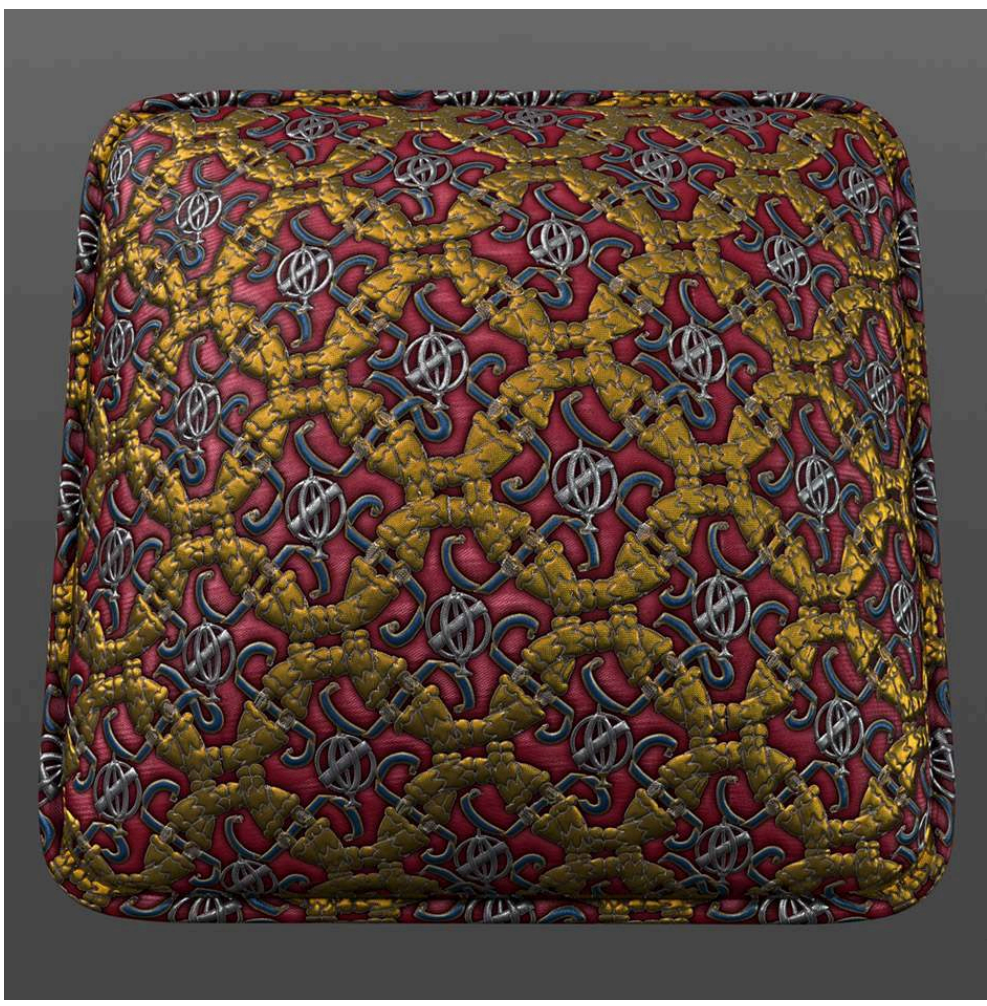

Essai de restitution des broderies d'après les décors sculptés du châlit. (c) Mazedia/Ludovic Gicqueau, 2016.

Figure 15

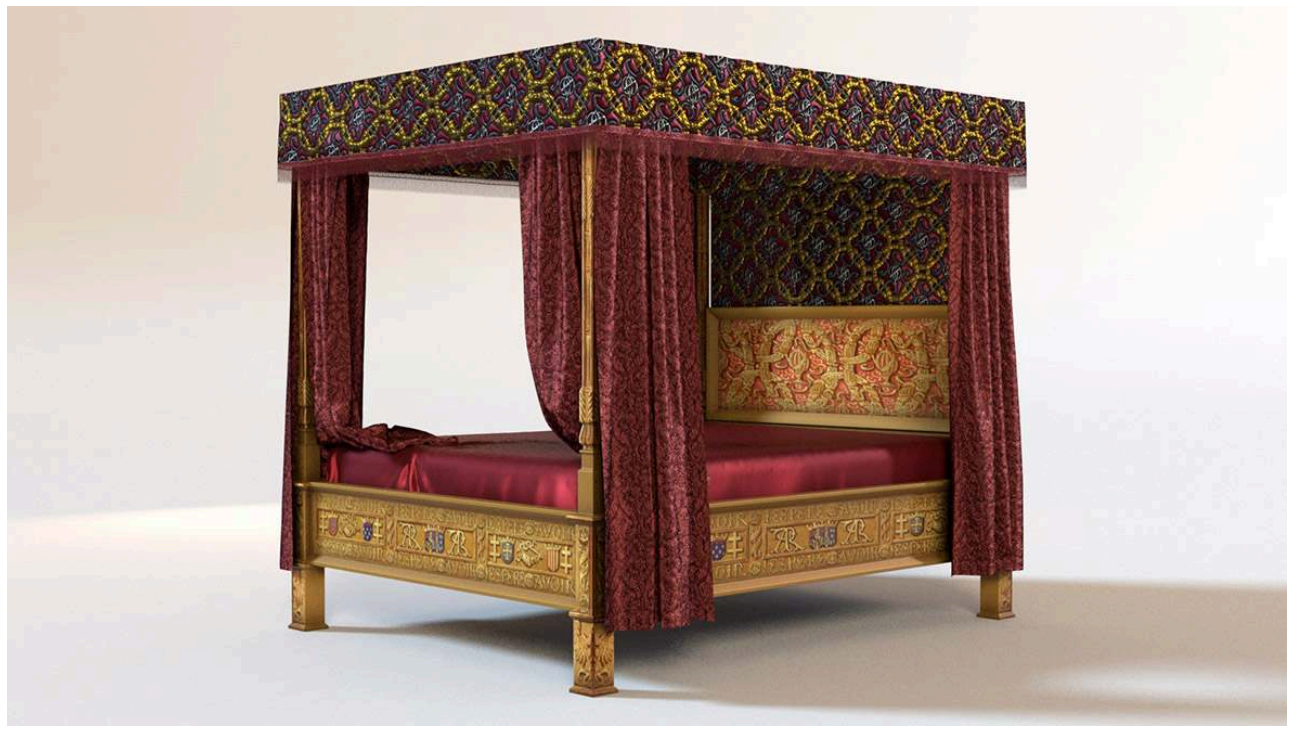

État de 1532 restitué.

(c) Palais des ducs de Lorraine-Musée lorrain/Mazedia, 2017. 


\section{L'état de 1543}

L'inventaire de 1543 décrit les parties textiles différemment :

- No 24 «Un ciel de lict de satin cramoisy, chargé de chappeaulx de triumphe et des espers de broderie de toille d'or et de toille d'argent »;

- No 25 « Trois rideaulx de damas blanc»;

- N 26 « Une contrepoincte de taffetas violet »;

- $\mathrm{N}^{\circ} 27$ « Une mante taincte en escarlatte $»^{36}$.

Nous en avons déduit que le ciel était resté le même qu'en 1532 ; l'inventaire de 1543 précise d'ailleurs que les chapeaux de triomphe et les sphères sont en broderies de toile d'or et d'argent ce qui signifie que ces motifs étaient réalisés en broderie d'application sur une base de toile d'or et toile d'argent ; " toile d'or » et " toile d'argent » sont pris ici pour "drap d'or » et «drap d'argent », termes appliqués aux textiles incorporant des fils d'or ou des fils d'argent sur une armure de fond en soie. Ces fils métalliques (rarement en or ou argent pur) étaient insérés sous forme de flottés de trame, jamais comme fils de chaîne ni comme trame de fond ${ }^{37}$. Ces applications étaient elles-mêmes brodées à l'aiguille. Il n'est plus fait mention du dosseret que nous avons néanmoins choisi de maintenir dans la restitution. En revanche, les rideaux sont clairement décrits en damas blanc; le modèle en a été pris dans ceux qui forment l'arrière-plan du Portrait de jeune homme à la lampe de Lorenzo Lotto (fig. 16), dont le motif et la datation concordaient.

\section{Figure 16}

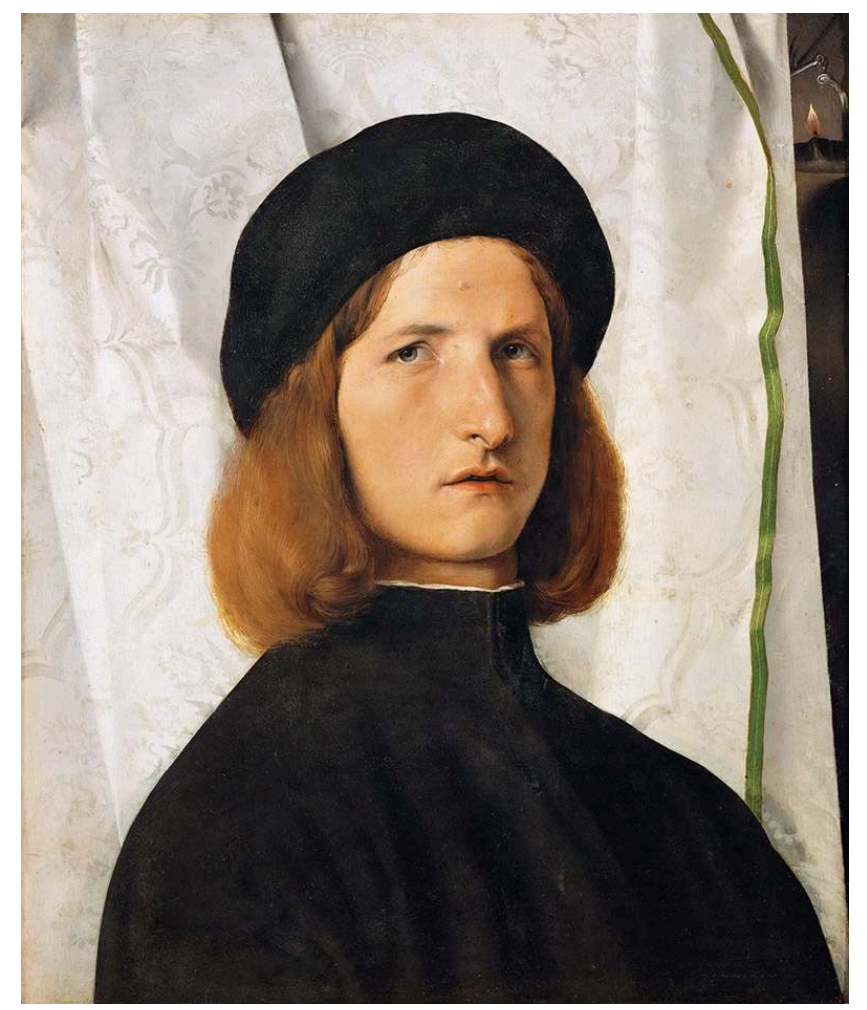

Lorenzo Lotto, Portrait de jeune homme à la lampe, 1506-1508, peinture à I'huile sur bois. Vienne, Kunsthistorisches Museum, GG_214.

Wikimedia Commons (No Copyright). 

généralement assortie au ciel, aux pentes et aux courtines, elle est ici en taffetas violet, c'est-à-dire une toile de soie. Il a fallu atténuer la brillance par rapport à l'état 1532 car le taffetas est une étoffe de soie mate; ses plis sont aussi moins souples et plus cassants. Le lit était alors aussi doté d'une "mante", pièce qui se pose sur la courtepointe comme un édredon. Elle est plus épaisse et ne retombe généralement pas sur les côtés. Elle est décrite comme étant de couleur écarlate, teinte que nous avons imaginée un peu plus soutenue que le cramoisi. L'armure textile n'étant pas précisée, nous avons retenu le taffetas, qui sert généralement pour cet élément. Les mantes sont piquées, c'est-à-dire parcourues de petites coutures qui permettent de maintenir leur rembourrage en place. Ces piqûres jouent aussi un rôle décoratif. Les informaticiens ont travaillé sur l'aspect moelleux et duveteux car ces mantes étaient en général garnies de plume (fig. 17).

Figure 17

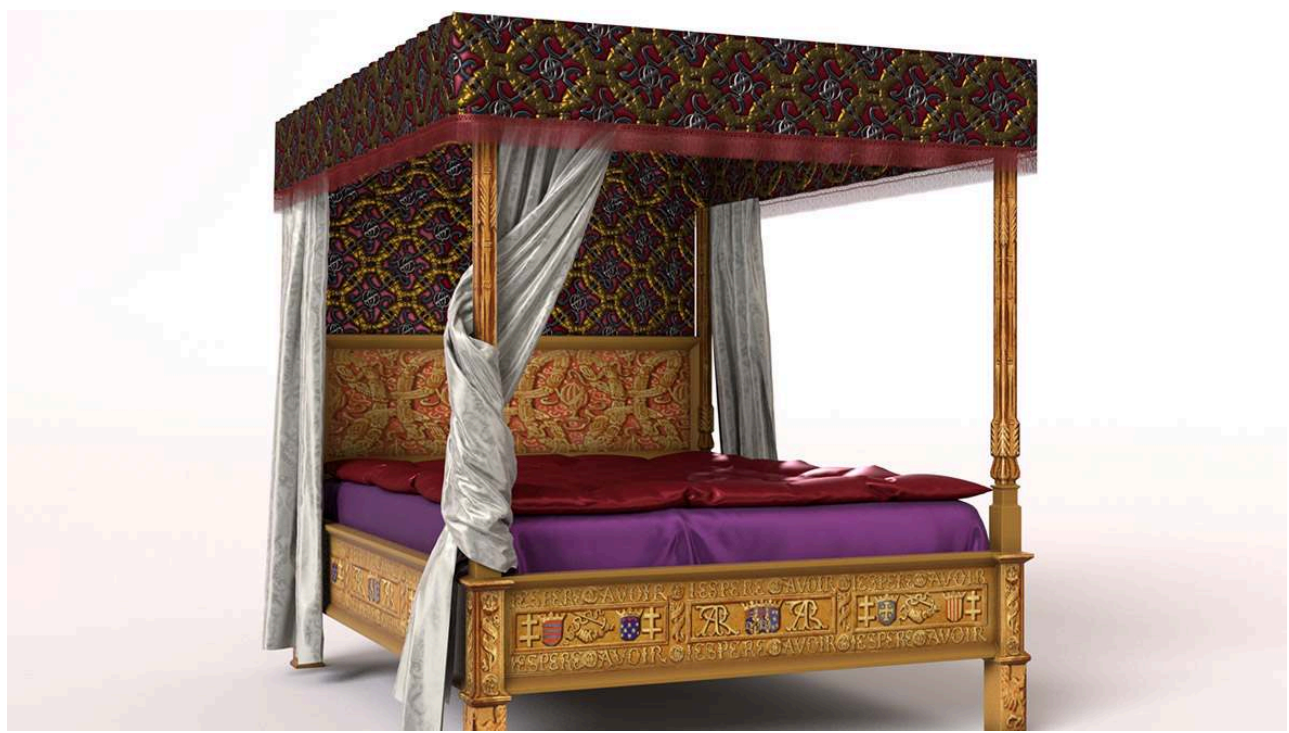

État de 1543 restitué.

(c) Palais des ducs de Lorraine-Musée lorrain/Mazedia, 2017.

\section{L'état de 1606}

L'inventaire dressé en 1606 révèle les modifications apportées à la parure du lit, probablement pour la mettre au goût du jour au début du XVII ${ }^{\mathrm{e}}$ siècle :

- No 114 «Un lict, satin cramoisy avec des chappeaux de triomphe faictz en fondz de broderie de toille d'argent filés aux devises de J'espère avoir, six pentes frangées de soie rouge aux crespines d'or nouées, le dict lit garny de son docier.

- No 115 Trois rideaux, damas cramoisy à un passement d'or à jour, sur les coutures frangées de soye rouge et d'or $»^{38}$.

Ciel, pentes et dosseret ressemblent à l'état de 1532. Le nombre des pentes (6) est donné pour la première fois; c'est cette mention qui nous a convaincus qu'il existait dès l'origine des pentes intérieures et extérieures. Les franges des pentes sont aussi mentionnées pour la première fois. En les comparant avec d'autres mentions de lits 
dans les sources, il nous a paru évident que les pentes étaient initialement pourvues de franges. Le fait qu'elles soient décrites ici indique probablement un changement expliqué par le choix de fils d'or associés aux fils de soie rouge. Il s'agit donc de franges plus sophistiquées qu'en 1532 et 1543. Leur modèle a été pris dans les passementeries du commerce en les modifiant pour s'approcher des franges et passementeries visibles sur les gravures d'Abraham Bosse. De nombreux essais ont été faits pour aboutir à ces « crépines d'or nouées » qu'il faut imaginer comme un large galon ajouré.

En revanche, les courtines ne sont alors plus de damas blanc. Au nombre de trois, elles sont en damas rouge et soulignées sur le grand côté visible et en partie basse par un galon et des franges. L'état des textures et des couleurs s'inspire du lit de Boughton House, château des ducs de Montagu, acquis par le Victoria and Albert Museum $^{39}$. Le damas est une étoffe de soie aux motifs ton sur ton dont le sens du tissage crée des effets de brillance opposés. Plusieurs pièces des collections du musée national de la Renaissance et du musée des Arts décoratifs de Paris ont servi de modèles pour ce tissu ${ }^{40}$. Sur le pourtour des rideaux, c'est-à-dire sur les côtés de l'ouverture (grand côté visible et partie basse) se trouvent des franges rouges et or mêlées (comme le long des pentes). Pour ces passementeries, des produits du commerce ont servi de modèle et ont été modifiés pour obtenir l'effet mêlé de soie rouge et de fils or ${ }^{41}$. De plus, le long des rideaux, cousu sur le damas, se trouve un galon de fils d'or pour lequel on s'est inspiré de galons appartenant à des ornements liturgiques conservés au musée national de la Renaissance ${ }^{42}$. Ce type de courtine à galon correspond effectivement à celles en usage au début du XVII ${ }^{e}$ siècle, dont de nombreux exemples sont visibles sur les gravures d'Abraham Bosse. Aucune indication nouvelle n'étant apportée quant à la courtepointe, il a été décidé de maintenir pour l'état 1606 celle de l'état de 1543 accompagnée de la mante (fig. 18).

Figure 18

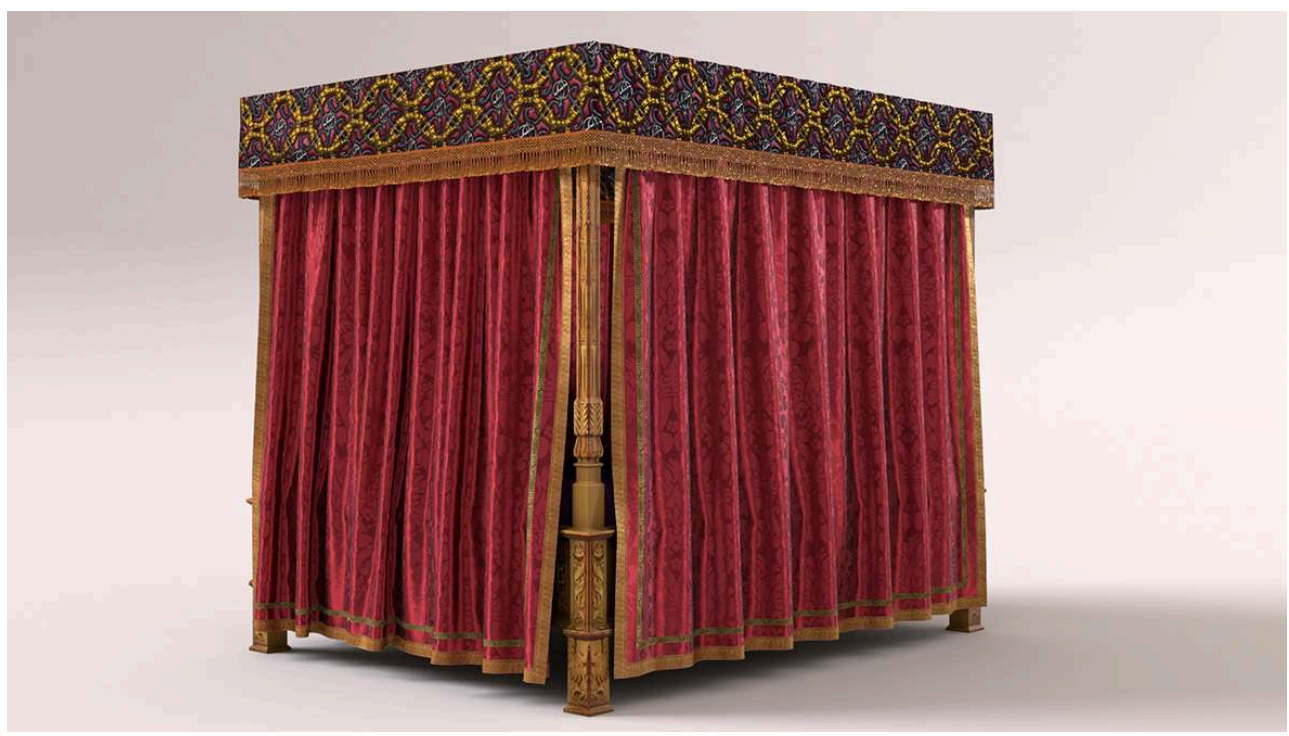

État de 1606 restitué.

(C) Palais des ducs de Lorraine-Musée lorrain/Mazedia, 2017. 


\section{Conclusion}

Ces trois restitutions virtuelles, comme les documents d'archives sur lesquels elles se fondent, révèlent l'aspect évolutif et non figé du textile dû à son usure mais aussi aux changements de mode, y compris pour un meuble insigne et emblématique d'une dynastie et d'un pouvoir comme le lit du duc Antoine et de la duchesse Renée de Bourbon. Pourtant, ces restitutions ne demeurent que des propositions en raison de l'imprécision des sources et du petit nombre d'étoffes de la Cour de Lorraine qui nous sont parvenues ${ }^{43}$.

Les outils multimédia comme celui-ci ouvrent de larges perspectives sur le plan de la médiation et permettent de rendre sa forme à une œuvre qui a perdu une partie de ce qui la constituait : le textile. Mais au-delà, ce travail entre graphistes-informaticiens et historiens de l'art est très stimulant et très enrichissant car il suscite des interrogations à propos de l'objet d'étude, interrogations qui n'auraient pas forcément été soulevées sans l'existence d'un tel projet.

\section{NOTES}

1. - JALABERT, Laurent et PÉNET, Pierre-Hippolyte. La Lorraine pour horizon. La France et les duchés. Milan : Silvana Editoriale, 2016, p. 34-35.

2. - VOLCYR, Nicolas. «Baptesme de Nicolas-Monsieur filz puis-nay de Monseigneur le duc Antoine, depuis comte de Vaudémont, duc de Mercœur, marquis de Nomeny, comte de Challigny : À Bar le X novembre M.D.XXIIII » (BnF, Réserve des livres rares, RES-4-LK2-3719, voir sur Gallica: https://gallica.bnf.fr/ark:/12148/btv1b55006572f/f11.image [consulté le 26/06/2019]). Publié par DIGOT, Auguste. Mémoires de la Société des sciences, lettres et arts de Nancy, 1848, p. 147-162, ici p. 157.

3. - Ces trois inventaires manuscrits issus de la collection de Lorraine, conservée à la Bibliothèque nationale de France, ont été publiés dans Recueil d'inventaires des ducs de Lorraine. Éd. Charles Guyot. Nancy : René Wiener, 1891, p. 24-35, 66-115 et 285-340.

4. - Pour quelques éléments biographiques, voir le site : http://cths.fr/an/savant.php?id=101601 [consulté le 26/06/2019].

5. - GUERRIER de DUMAST, Auguste Prosper François. Nancy, Histoire et tableau. Nancy : Conty, 1837, p. 310.

6. - Pour quelques éléments biographiques, voir le site : http://cths.fr/an/savant.php?id=102543 [consulté le 26/06/2019].

7. - SAMOYAULT, Jean-Pierre. Lit du duc Antoine de Lorraine et de la duchesse Renée de Bourbon-Montpensier. Dans CRÉPIN-LEBLOND, Thierry (dir.). Parures d'or et de pourpre. Le mobilier à la cour des Valois. Cat. exp. Blois, château de Blois, 15 juin-30 septembre 2002. Paris: Somogy éditions d'art, 2002, p. 79.

8. - Arch. nat., $\mathrm{O}^{4} 1638 \mathrm{~B}$, cité par SAMOYAULT, Jean-Pierre. Ibid., p. 81.

9. - Arch. nat., AJ 19 633, idem.

10. - Lettre du maréchal Vaillant, ministre de la maison de l'Empereur et des Beaux-Arts, Journal de la Société d'archéologie et du Comité du Musée lorrain, juillet 1864, p. 136. 
11. - Arch. nat., AJ 19644 , idem.

12. - Sur cette société, voir le site : http://cths.fr/an/societe.php?id=1495\&proso=y\&soc_liees= [consulté le 26/06/2019].

13. - En 1864, le maréchal Vaillant explique ainsi son refus d'accéder à la demande des membres du Comité du Musée lorrain : «Ce meuble présente un véritable intérêt, comme spécimen de l'art français du XVI ${ }^{\mathrm{e}}$ siècle, et l'abandon qui en serait fait au Musée lorrain serait d'autant plus regrettable pour le garde-meuble de la Couronne, que, par suite des ventes faites dans les palais, en 1792 et en 1793, il ne possède plus qu'un très petit nombre de meubles artistiques remontant au-delà du règne de Louis XVI ». Journal de la Société d'archéologie et du Comité du Musée lorrain, juillet 1864, p. 136.

14. - L'architecte Charles-François Chatelain (1802-1873) offre au musée en 1863 un dessin du « lit du duc Antoine ». Peut-être s'agit-il de celui que nous évoquons. Journal de la Société d'archéologie et du Comité du Musée lorrain, mars 1863, p. 54.

15. - Journal de la Société d'archéologie et du Comité du Musée lorrain, juillet 1852, p. 74, cité par J.-P. SAMOYAULT. Op. cit.

16. - L'embryon de musée installé dans la galerie des Cerfs en 1850 est ravagé par un incendie le 17 juillet 1871. Deux architectes, Émile Boeswillwald et Prosper Morey, sont chargés conjointement de la restauration du bâtiment.

17. - Rappelons que suite au traité de Vienne de 1738, la famille de Lorraine cède ses États au roi Stanislas Leszczynski puis, à son décès, à la Couronne de France. Le dernier duc de la maison de Lorraine, François III, devient en échange grand-duc de Toscane avant d'être élu empereur en 1745. Le mobilier des résidences ducales est transféré à Florence puis à Vienne où peu d'éléments d'origine lorraine ont pu être identifiés à ce jour.

18. - Au sujet des armes et emblèmes ducaux, on pourra lire « Le blason et les emblèmes du duc René II » dans JALABERT, Laurent et PÉNET, Pierre-Hippolyte. Op. cit., p. 25-28.

19. - Sur l'étude de ces devises, nous renvoyons à l'ouvrage majeur : CHONÉ, Paulette. Emblèmes et pensée symbolique en Lorraine (1525-1633). Paris : Klincksieck, 1991, p. 85-108.

20. - CHRISTIN, Olivier (dir.). Un nouveau monde. Naissance de la Lorraine moderne. Cat. exp. Nancy, Musée Lorrain, 4 mai-4 août 2013. Paris/Nancy : Somogy éditions d'art/Musée lorrain, 2013.

21. - Voir ci-dessous.

22. - Ce comité scientifique était composé de Muriel Barbier (musée national de la Renaissance), Magali Bélime-Droguet (CMN), Roberta Cortopassi (C2RMF), Richard Dagorne (Musée lorrain) et Jean-Jacques Gautier (Mobilier national). Le projet était suivi par Frédérique Gaujacq (Musée lorrain).

23. - Restaurateur mobilier : Aubert Gérard; tapissier : Michel Chauveau; tissu : Le Manach.

24. - La réalisation de ce dispositif numérique est le fruit d'un partenariat entre le Palais des ducs de Lorraine-Musée lorrain et le musée national de la Renaissance. Dans le cadre de l'opération «Le musée sort de son palais », organisée durant la fermeture du palais ducal à l'occasion des travaux de rénovation, le lit est présenté au musée national de la Renaissance depuis le mois d'avril 2017 avec son dispositif de médiation.

25. - Par voie électronique et lors de la séance de travail qui s'est tenue au musée national de la Renaissance le 15 juin 2017.

26. - Voir ci-dessous.

27. - SCNHEEBALG-PERELMAN, Sophie. «Richesse du garde-meuble parisien de François I ${ }^{\mathrm{er}}$. Inventaires inédits de 1542 et $1551 »$. Gazette des beaux-arts, 1971, II, p. 253-304.

28. - Voir dans ce numéro : BARBIER, Muriel. « Le vocabulaire des lits de la Renaissance en France à la lumière des sources ", In Situ [En ligne], 40 | 2019, mis en ligne le 10 septembre 2019, consulté le 26 septembre 2019. URL : http://journals.openedition.org/insitu/23340. 
29. - Ce tableau a aussi servi de référence pour la restitution du lit de Philippe Lesbahy au château d'Azay-le-Rideau; voir BÉLIME-DROGUET, Magali. " "Une chambre bien nattée": la chambre de Philippe Lesbahy à Azay-le-Rideau ». Dossier de l'Art, n² 204, 2013, p. 94-96.

30. - L'expertise de Xavier Bonnet ainsi que la connaissance des sources par Monique Chatenet ont été d'un grand secours dans ces débats.

31. - Sur les questions des effets de la lumière sur le textile, voir LECLERCQ, Jean-Paul. Jouer la lumière. Cat. exp. Paris, musée de la Mode et du Textile, 25 janvier 2001-janvier 2002. Paris : Adam Biro/UCAD, 2001.

32. - Paris, BnF, Ms 462 (1532), Inventaire des biens conservés à la galerie des armoires du palais ducal, publié dans Recueil d'inventaires des ducs de Lorraine. Op. cit., p. 28 et p. 31.

33. - Pour les précisions de vocabulaire, voir dans ce numéro : BARBIER, Muriel. «Le vocabulaire des lits de la Renaissance en France à la lumière des sources ", In Situ [En ligne], 40 | 2019, mis en ligne le 10 septembre 2019, consulté le 26 septembre 2019. URL : http://journals.openedition.org/ insitu/23340 ; et COURTIN, Nicolas. "Le vocabulaire et les typologies des lits à Paris d'après les inventaires après décès de la première moitié du XVII ${ }^{\mathrm{e}}$ siècle ", In Situ [En ligne], 40 |2019, mis en ligne le 15 septembre 2019, consulté le 26 septembre 2019. URL : http://journals.openedition.org/ insitu/23316.

34. - BARBIER, Muriel. «Quelles étoffes pour les lits des grandes occasions dans la France de la Renaissance?». Dans COUPRY, Claude et COUSIN, Françoise (dir.). Étoffes des grands jours. Paris : AFET, 2018, p. 36.

35. - VOLCYR, Nicolas. Op. cit..

36. - Paris, BnF, Ms 462 (1543), Inventaire des biens étant dans la galerie et au rond du palais ducal, publié dans Recueil d'inventaires des ducs de Lorraine. Op. cit., p. 70.

37. - MONNAS, Lisa. Merchants, Princes and Painters, Silk Fabrics in Italian and Northern Paintings 1300-1550. New Haven/London : Yale University Press, 2008, p. 299 ; BARBIER, Muriel. Art. cit., note 25, p. 33.

38. - Paris, BnF, Ms 462 (1606), "Déclaration est estimation des "bagues et joyaux" et des "meubles précieux" de [sic] Son Altesse veult et entend estre unis incorporez, joincts et demeurer à tousjours au duché de Lorraine ", publié dans Recueil d'inventaires des ducs de Lorraine. Op. cit., p. 304.

39. - Voir le site: http://collections.vam.ac.uk/item/O81989/boughton-bed-bed-unknown/ [consulté le 26/06/2019] : ce lit, bien que plus récent (il date des années 1670-1680), est recouvert d'un damas cramoisi et de galons or dont l'effet a servi d'exemple.

40. - En particulier, une robe de magistrat vénitien en damas datée du début du XVII siècle (Paris, musée des Arts décoratifs, inv. 7858) ; voir LECLERCQ, Jean-Paul. Op. cit. note 22, p. 172-173.

41. - Les fournisseurs de passementeries consultés sont Carlhian (Lyon) et Houlès (Paris).

42. - Atelier français, chasuble, $\mathrm{xVI}^{\mathrm{e}}$ siècle, orfrois brodés sur velours coupé, Écouen, musée national de la Renaissance, E. Cl. 1224.

43. - Pour visualiser l'intégralité de l'outil multimédia, voir le site: https://www.museelorrain.nancy.fr/fr/collections/les-oeuvres-majeures/lit-du-duc-antoine-de-lorraine-et-derenee-de-bourbon-64 [consulté le 26/06/2019]. 


\section{RÉSUMÉS}

Exécuté à l'occasion du mariage du duc Antoine de Lorraine et de Renée de BourbonMontpensier, en 1515 , le lit des époux, bien que modifié au xIX ${ }^{\mathrm{e}}$ siècle, est l'un des plus anciens conservés en France. Il est aussi porteur d'une riche symbolique associant les emblèmes et devises de la Maison de Lorraine aux monogrammes entrelacés des deux époux. En vue de sa présentation lors de l'exposition « Un Monde nouveau» (Nancy, Musée lorrain, 2013), ce lit a reçu une nouvelle garniture textile, opération qui a donné l'occasion de relire les trois inventaires des ducs de Lorraine conservés pour la période (1532, 1543 et 1606). Cette étude a abouti à une meilleure compréhension des étoffes et à la création d'un multimédia pédagogique créé en concertation avec le Comité lit. Cette restitution virtuelle permet de comprendre l'évolution de la parure textile et d'en montrer les trois états connus. C'est le résultat de ces recherches qui est ici présenté.

Made in 1515 for the marriage of Duke Antoine de Lorraine and Renée de Bourbon-Montpensier, the bed of this married couple, although modified during the nineteenth century, is one of the oldest beds preserved in France today. It has a rich symbolic decoration associating the emblems and mottos of the House of Lorraine with the intertwined monograms of the two spouses. On the occasion of its presentation at the exhibition Un Monde nouveau (Musée Lorrain, Nancy, in 2013), this bed was given a new textile upholstery. This presented an opportunity to study anew the three inventories of the Dukes of Lorraine, preserved for this period and the years1532, 1543 and 1606. The study resulted in a better understanding of the textiles present and the creation of an educational multimedia tool, developed in consultation with the Bed Committee. It is a virtual restitution which makes it possible to understand the evolution of the textile elements of the bed and to show the bed's three known states. The results of this research are presented in this present article.

\section{INDEX}

Keywords : bed, Antoine de Lorraine, Renée de Bourbon-Montpensier, museum, Musée Lorrain, Renaissance, furniture, chamber, Lorraine, textile, marriage, avalerion, drawstring, triumph hat, sphere

Mots-clés : lit, Antoine de Lorraine, Renée de Bourbon-Montpensier, musée, Musée lorrain, Renaissance, mobilier, chambre, Lorraine, textile, mariage, alérion, cordelière, chapeau de triomphe, sphère

\section{AUTEURS}

\section{MURIEL BARBIER}

Conservateur du patrimoine, Mobilier national muriel.barbier@culture.gouv.fr

\section{PIERRE-HIPPOLYTE PÉNET}

Conservateur du patrimoine, Nancy, Palais des ducs de Lorraine-Musée lorrain PierreHippolyte.Penet@mairie-nancy.fr 